\title{
On Spurious Interactions among a Mixed Layer Model, Convective Adjustment, and Isopycnal Mixing in Ocean Circulation Models
}

\author{
ANDREAS OSCHLIES* \\ Centre National de la Recherche Scientifique, Toulouse, France
}

17 July 1998 and 30 September 1998

\begin{abstract}
Inconsistencies can arise in ocean circulation models when part of the physical processes responsible for vertical mixing is described in the usual differential form and part is formulated as adjustment processes. Examples for the latter class are explicit convective adjustment and Kraus-Turner type models of the surface mixed layer. Implicit convective adjustment as well as various representations of interior-ocean mixing are normally described in differential form. All these schemes mix density, with a mixing intensity that itself depends on stratification. This requires that information concerning static stability is passed through the individual mixing routines in a consistent sequence. It is shown that inconsistencies can arise when coupling a Kraus-Turner type model of wind-induced mixing with both a standard implicit convective adjustment as well as with an isopycnal mixing scheme. This leads to considerably overestimated mixed layer depths, for example, by hundreds of meters in the subpolar North Atlantic. The problem is eliminated first by ensuring that dissipation of potential energy during convection is included in the mixing scheme, even when considering wind-induced turbulence only, and second, by either calling the mixed layer routine before the differential vertical mixing scheme or tapering the vertical diffusivities to zero within the surface mixed layer.
\end{abstract}

\section{Introduction}

Hydrostatic ocean circulation models like the Geophysical Fluid Dynamics Laboratory (GFDL) Modular Ocean Model (MOM; Pacanowski et al. 1991) have to employ some sort of convective adjustment in order to remove statically unstable stratification. Two alternative types of convective adjustment are standard options in the GFDL MOM. The first method, called explicit convective adjustment, mixes vertically adjacent grid boxes if they are found to be unstable. Mixing is instantaneous and complete, and does not need the intermediate computation of diffusivities. The second method, called implicit convection, parameterizes convective overturning by increasing the coefficient for vertical diffusion, which enters the differential representation of vertical mixing. To overcome the time step constraint associated with the large vertical diffusivities, vertical mixing is then computed implicitly. Similarly, implicit vertical mixing is the standard method when using isopycnal

* Current affiliation: Institut für Meereskunde an der Universität Kiel, Kiel, Germany.

Corresponding author address: Dr. Andreas Oschlies, Institut für Meereskunde an der Universität Kiel, Düsternbrooker Weg 20, 24105 Kiel, Germany.

E-mail: aoschlies@ifm.uni-kiel.de mixing, which increases the coefficient for vertical diffusion with increasing slope of isopycnal surfaces. All of the above schemes calculate mixing intensity as a function of static stability. Static stability can, however, also be modified by the action of a mixed layer model, which, in turn, depends on the density field. Special care therefore has to be taken to ensure that information concerning stratification is passed through the individual subroutines in a consistent sequence. This is demonstrated with a series of experiments using the World Ocean Circulation Experiment (WOCE) Community Modeling Effort (CME) model of the North Atlantic Ocean (Bryan and Holland 1989). It is shown that inconsistencies present in some previous CME experiments that used a simple Kraus-Turner type representation (Kraus and Turner 1967) of wind-forced mixing in the oceanic surface boundary layer (e.g., Böning and Herrmann 1994; Oschlies and Willebrand 1996) produced overly deep winter mixed layers in large parts of the model domain. The circulation model, convective adjustment schemes, and mixed layer model are briefly described in the following section. In section 3 , the spurious interactions caused by an inconsistent coupling of mixed layer physics and convective adjustment as well as isopycnal mixing are investigated and a solution to the problem is given. The paper ends with a brief discussion of the relevance to previously published modeling studies. 


\section{Model physics}

\section{a. The circulation model}

The CME model of the North Atlantic is used in its high-resolution version with a grid spacing of $1 / 3^{\circ}$ in meridional and $25^{\circ}$ in zonal direction. The number of vertical levels has been increased from 30 in the standard version (Bryan and Holland 1989) to 37, with all seven additional levels being added in the upper $150 \mathrm{~m}$ (Oschlies and Garçon 1999). The finer vertical resolution was introduced to prepare for coupling with a simple ecosystem model. It is not vital for the results presented below. No attempt is made to resolve high-frequency forcing or the daily cycle, and the model is forced with climatological datasets. Monthly mean wind stresses, $\langle\tau\rangle$, as well as the monthly mean of the third power of the friction velocity, $\left\langle u_{*}^{3}\right\rangle$, where $u_{*}=(|\tau| /$ $\left.\rho_{w}\right)^{1 / 2}$ in water of density $\rho_{w}$, are taken from Hellerman and Rosenstein (1983). The thermohaline forcing is represented by a relaxation of surface salinity to the monthly mean values of Levitus (1982) and a heat flux given by the linear formulation of Han (1984). Subgridscale mixing is accounted for by the highly scale-selective biharmonic operator in the horizontal, while a standard second-order parameterization for diffusion and viscosity is used in the vertical, with constant coefficients, $K_{\rho}$ $=0.3 \mathrm{~cm}^{2} \mathrm{~s}^{-1}$ and $K_{m}=10 \mathrm{~cm}^{2} \mathrm{~s}^{-1}$, respectively. For conceptual simplicity, penetration of solar radiation is not taken into account here.

The GFDL MOM code principally offers two different options to remove static instabilities by convective adjustment:

- Explicit convection: At the end of each time step the water column is scanned and unstable parts of the water column are homogenized. Here we shall use the method of Rahmstorf (1993). In contrast to the original GFDL convection scheme the new algorithm guarantees complete removal of all static instability whithin one time step (e.g., Marotzke 1991). In a schematic way, the algorithm can be written as

$$
T^{t+\Delta t}=T^{*}+\operatorname{CA}\left(T^{*}, S^{*}\right),
$$

where $T^{*}, S^{*}$ are temperature and salinity at time step $t+\Delta t$ after all tendency terms (e.g., surface fluxes) have been added, but before the convective adjustment scheme (CA) has been applied. A corresponding treatment is applied to the salinity field.

- Implicit convection: This option was developed by M. Cox as an alternative to the the original GFDL convection scheme. It treats convective overturning as vertical diffusion, by setting the vertical diffusion coefficient to a very high value $\left(10^{6} \mathrm{~cm}^{2} \mathrm{~s}^{-1}\right.$ in the present model; test experiments with $10^{4} \mathrm{~cm}^{2} \mathrm{~s}^{-1}$ did not show significant differences) in cases of static instability. For numerical stability reasons associated with the large diffusivities, vertical diffusion is calculated implicitly by solving

$$
T^{t+\Delta t}=T^{*}+2 \Delta t \frac{\partial}{\partial z}\left(K_{\rho}\left(\rho^{t-\Delta t}\right) \frac{\partial T^{t+\Delta t}}{\partial z}\right) .
$$

A time step $2 \Delta t$ is used to match the leapfrog time step used in the advection terms. The vertical diffusivity $K_{\rho}$ is a function of the density field $\rho^{t-\Delta t}=$ $\rho\left(T^{t-\Delta t}, S^{t-\Delta t}\right)$ at the previous time step $t-\Delta t$. Essentially, the same differential form of diffusion is used for the vertical component of isopycnal mixing (e.g., Pacanowski et al. 1991).

It is important to note that the vertical diffusivity $K_{\rho}$ is a function of the density field at a previous time step. [Using $K_{\rho}\left(\rho^{t}\right)$ instead of $K_{\rho}\left(\rho^{t-\Delta t}\right)$ in the implicit convection algorithm was found to work equally well.] This is in contrast to the explicit convective adjustment (1) and also to the Kraus-Turner type mixed layer model described below. Although it may in principle be possible to construct the differential form of vertical diffusion using $K_{\rho}\left(\rho^{*}\right)$ with $\rho^{*}=\rho\left(T^{*}, S^{*}\right)$, this has-to the author's knowledge-never been attempted. Problems associated with using $K_{\rho}\left(\rho^{*}\right)$ include cases where a variable $K_{\rho}$ is applied also to other variables (e.g., momentum, biological tracers) that are stepped forward in time before calling the tracer routine, that is, before $\rho^{*}$ is known. Even more prohibitive is the use of $K_{\rho}\left(\rho^{*}\right)$ for isopycnal diffusion, as the necessary information about horizontal density gradients would require knowledge of $\rho^{*}$ at surrounding gridpoint columns. It is, however, not the intention of this paper to develop new numerical schemes, but to draw attention to the careful combinations of vertical mixing routines in existing, generally well-working models.

In the following we shall concentrate on the common differential form (2) of implicit convection. The extent of convective activity at time step $t+\Delta t$ depends on the static situation after convection at a previous time step. Interestingly, this does not, in general, present a problem because the implicit convection scheme does not completely remove static instabilities. Although vertical diffusivities will be set to a very high value, diffusion can only asymptotically homogenize the statically unstable water column. The remaining instabilities observed in the model (where $2 \Delta t=1 \mathrm{~h}$ and the $e$ folding mixing timescale for a 100-m thick column and $K_{\rho}=10^{6} \mathrm{~cm}^{2} \mathrm{~s}^{-1}$ is $100 \mathrm{~s}$ ) are, however, negligibly small. Without the action of other processes (horizontal density fluxes, surface forcing) the sign of the static stability, $\partial \rho / \partial z$, will be conserved in regions of constant $K_{\rho}$. On the other hand, stabilizing density changes over one time step (e.g., surface warming in spring) usually suffice to maintain a stable (though very weak) stratification after the intermediate $\rho^{*}$ field has been vertically diffused by the implicit convection scheme. With $\partial \rho^{t+\Delta t} / \partial z<0$ there will then be no convection in the subsequent time step. Note that the explicit convective adjustment would terminate convection already for $\partial \rho^{*} / \partial z<0$. For situations typically encountered in the 
present model configuration with monthly mean forcing the implicit scheme can be viewed as lagging the explicit convection by one time step.

Both of the above convective adjustment schemes have been used in the CME model described above and were found to produce essentially identical results under seasonal forcing, as long as no additional mixed layer routine was applied. Marked differences did, however, appear when the Kraus-Turner type mixed layer described below was embedded in the circulation model in the apparently natural way that has been used in some previous CME experiments.

\section{b. A Kraus-Turner type mixed layer model}

Kraus-Turner type models (Kraus and Turner 1967) treat the surface mixed layer as a homogeneous slab, its depth $h$ being determined from a balance equation for the vertically integrated budget of turbulent kinetic energy (TKE). Tracers are homogenized down to the depth of the surface mixed layer in an adjustment process similar to the explicit formulation of convection. A slightly simplified form of the vertically integrated, steady state TKE equation reads (e.g., Niiler and Kraus 1977)

$$
\frac{1}{2} w_{e} \Delta b h=m u_{*}^{3}+\frac{h}{4}\left[(1+n) B_{0}-(1-n)\left|B_{0}\right|\right],
$$

where $w_{e}$ is the entrainment velocity and $\Delta b$ the jump of specific buoyancy, $b=-g\left(\rho-\rho_{0}\right) / \rho_{0}$, across the base of the mixed layer. Mechanical production of TKE is parameterized by the first term on the right-hand side as being proportional to the wind forcing, that is, the cube of the friction velocity (in the water), $u_{*}^{3}$. Not explicitly included is TKE production by vertical shear. Sterl and Kattenberg (1994) found that TKE production by vertical shear simulated by an ocean circulation model was, to a good approximation, proportional to the cube of the friction velocity and may therefore be included in the $u_{*}^{3}$ term. It is, however, not evident how one can adequately simulate TKE production through shear instabilities in present general circulation models that do not resolve the observed vertical shear spectrum down to the cutoff length of $O(\sim 10 \mathrm{~m})$ (e.g., Gargett 1986). The last term on the right describes the TKE gain or loss that results from the action of surface buoyancy fluxes $B_{0}$. If $B_{0}>0$ (e.g., cooling) TKE is produced, of which the fraction $(1-n)$ is dissipated within the mixed layer. The constants $m$ and $n$ are adjustable parameters.

Following Camp and Elsberry (1978), the TKE that remains after dissipation of the TKE originally produced through wind forcing may be assumed to decrease exponentially with depth, such that

$$
m=m_{1} e^{-h / D} \text {. }
$$

In this study the empirically optimized parameter values $m_{1}=0.3$ and $D=50 \mathrm{~m}$ were used throughout. [For reference, we note that $m_{1}$ is related to the mixing parameter $\alpha$ cited in earlier CME publications (e.g., Böning and Herrmann 1994) by $m_{1} \simeq 10^{3} \alpha$.]

An algorithm suitable for solving the TKE balance (3) for the mixing depth $h$ on the discrete vertical grid of a $z$-coordinate ocean circulation model was first introduced by Thompson (1976). For each depth level $z_{k}$ with layer thickness $\Delta z_{k}$ and the index $k$ increasing downward, the TKE budget is computed:

$$
\begin{aligned}
\Delta \mathrm{TKE}_{k} \equiv & 2 \Delta t \rho_{0} m_{1} u_{*}^{3} e^{-h^{\text {old } / D}} \\
& +\sum_{i=1}^{k} \frac{1}{2}\left[(n+1) \Delta P_{i}+(n-1)\left|\Delta P_{i}\right|\right],
\end{aligned}
$$

where

$$
\Delta P_{i}=-g \sum_{l=1}^{k_{\max }}\left(\rho_{l}^{i-1}-\rho_{l}^{i}\right) z_{l} \Delta z_{l},
$$

$\rho_{l}^{i}$ represents the density of layer $l$ when beginning from the surface layers $1, \ldots, i$ are mixed and layers $i+1$, $\ldots, k_{\max }$ are yet unaffected by the mixing. Here, $2 \Delta t$ is the leapfrog time step, and $h^{\text {old }}$ is the average (to suppress $2 \Delta t$ noise) mixed layer depth from time steps $t$ and $t-\Delta t$. If $\Delta P_{i}>0$ potential energy is lost by the mixing, of which the portion $n$ becomes TKE available to deepen the mixed layer. For $\Delta P_{i}<0$ mixing would increase the potential energy of the water column. If there exists some level $k$ with $\Delta \mathrm{TKE}_{k-1} \geq 0$ and $\Delta \mathrm{TKE}_{k}<0$ then the new mixing depth $h^{\text {new }}$ is determined by linear interpolation:

$$
h^{\text {new }}=z_{k-1}+\frac{\Delta \mathrm{TKE}_{k-1}}{\Delta \mathrm{TKE}_{k-1}-\Delta \mathrm{TKE}_{k}} \Delta z_{k} .
$$

If no such $k$ can be found the entire water column down to $z_{k \max }$ is homogenized.

While the above formulation is quite general, we now briefly illustrate its specific implementation in the CME model. Here, the Kraus-Turner routine is called at the end of each time step after the convective adjustment has taken place. It acts on the convectively adjusted $T^{t+\Delta t}, S^{t+\Delta t}$ fields of Eqs. (1) or (2), respectively. This reflects the intention of letting the mixed layer model account exclusively for wind-induced mixing (e.g., Böning and Herrmann 1994). With no static instabilities present (the slight instabilities that are left by the implicit convection scheme can safely be neglected in the potential energy calculation) we have $\Delta P_{i} \leq 0$, and mixing downward from the surface will always tend to increase the potential energy of the water column. Terms that contain the parameter $n$ cancel, and the TKE balance (5) reduces to

$$
\Delta \mathrm{TKE}_{k} \equiv 2 \Delta t \rho_{0} m_{1} u_{*}^{3} e^{-h^{\text {old }} / D}+E_{\mathrm{pot}}(0)-E_{\mathrm{pot}}(k),
$$

where

$$
E_{\mathrm{pot}}(0)=-g \sum_{l=1}^{k_{\max }} \rho_{l}^{0} z_{l} \Delta z_{l}
$$


refers to the potential energy of the water column just before calling the mixed layer routine, and

$$
E_{\mathrm{pot}}(k)=-g \sum_{l=1}^{k_{\max }} \rho_{l}^{k} z_{l} \Delta z_{l}
$$

is the potential energy that would result from homogenizing the uppermost $k$ grid boxes. Assuming that the mixed layer model accounts exclusively for wind-induced mixing, $k_{\max }$ was chosen such that $z_{\text {max }}=720 \mathrm{~m}$ in order to save computer time. Computational economy is also the motivation in replacing the original form (5) of the TKE budget by (8), which avoids the costly computation of the $\Delta P_{i}$.

We emphasize that the above argumentation depends crucially on the assumption that the convection scheme has effectively removed any contributions $\Delta P_{i}>0$ in (5). It will be shown below that this assumption does not always hold. If there are terms with $\Delta P_{i}>0$ then the use of (8) in fact corresponds to a parameter choice $n=1$ in the original TKE balance (5). In contrast to the intended modeling of wind-forced mixing, the mixed layer routine will then also convert the $\Delta P_{i}>0$ contributions into TKE, without any dissipation, and-as first discussed by Gill and Turner (1976) — simulate fully penetrative convection.

\section{Experiments}

The results of different 1-yr simulations will be presented. All experiments use identical forcing and the same initial conditions (taken from the end of a 24-yr integration of the CME model without mixed layer model), but different combinations of mixed layer and convective adjustment subroutines. The individual experiments are described as follows:

- NOIMP: no mixed layer, implicit convection (virtually indistinguishable from the corresponding experiment NOEXP with explicit convection).

- KTEXP: Kraus-Turner type wind-forced mixed layer, called after explicit convection.

- KTIMP: Kraus-Turner type wind-forced mixed layer, called after implicit convection; $K_{\rho}=10^{6} \mathrm{~cm}^{2} \mathrm{~s}^{-1}$ if $\partial \rho / \partial z>0$ as described in section $2 \mathrm{a}$. This is the standard version used in previous CME experiments with a mixed layer.

- KTISO: Kraus-Turner type wind-forced mixed layer, called after implicit convection. Convection already for neutrally stable situations; $K_{\rho}=10^{6} \mathrm{~cm}^{2} \mathrm{~s}^{-1}$ if $\partial \rho / \partial z \geq 0$.

\section{a. Mixed layer model and convection scheme}

\section{1) Problem}

Figure 1 shows the evolution of near-surface temperature at $30^{\circ} \mathrm{N}, 20^{\circ} \mathrm{W}$ over the 1 -yr integration period. Displayed in Fig. 1a is the annual cycle corresponding to experiment NOIMP. Winter mixed layer depths of about $140 \mathrm{~m}$ are followed by a very shallow summer mixed layer, essentially restricted to the first layer of the model $\left(\Delta z_{1}=11 \mathrm{~m}\right)$. The downward diffusion of heat in spring and summer is entirely due to the constant background diffusivity $K_{\rho}=0.3 \mathrm{~cm}^{2} \mathrm{~s}^{-1}$. Quite generally, spring and summer mixed layers would be slightly deeper if penetration of solar radiation were taken into account (e.g., Kraus and Rooth 1961).

Figure $1 \mathrm{~b}$ shows the results of experiment KTEXP. At each time step the explicit convective adjustment acts on the density field $\rho^{*}$ after all tendency terms have been added [Eq. (1)], leaving a convectively adjusted water column with all static instabilities removed. Only then the mixed layer model is called to further deepen the homogeneous surface layer. Due to the exponential decay of available wind-generated TKE [Eq. (4)] with an $e$-folding depth scale of $50 \mathrm{~m}$, the effect of wind stirring on the deep winter mixed layer is small.

In contrast to experiments without the mixed layer model, changing the convection scheme from explicit (Fig. 1b) to implicit (Fig. 1c) now produces a surprisingly different evolution of upper-ocean temperature. Differences are most pronounced in winter and autumn, with much greater mixed layer depths for the implicit convection experiment KTIMP. It was further found that there was essentially no convective adjustment taking place within the mixed layer. The mixed layer routine, which is called at the end of each time step, produces a homogeneous surface layer with $\partial \rho^{t+\Delta t} / \partial z=0$, thereby turning off implicit convection in the following time step. Obviously, this becomes a problem when the ocean loses buoyancy. A significantly unstable $\rho^{*}$ field enters the mixed layer model, and using Eq. (8) instead of (5) automatically implies the parameter choice $n=1$. All the TKE produced by buoyancy forcing will then be available for deepening the mixed layer. Quite in contrast to the original intention of modeling wind-forced mixing, the mixed layer model in experiment KTIMP is, in fact, simulating fully penetrative convection.

The impact of using configuration KTIMP rather than KTEXP over integration times of a few years typical for most previous CME experiments is demonstrated by Fig. 2. It shows the depth of the winter mixed layer after $5 \mathrm{yr}$ of integration for KTEXP and KTIMP, respectively. Almost everywhere, the mixed layer simulated by experiment KTIMP is considerably deeper than that of KTEXP. This increase of the winter mixed layer depth for a mixing parameterization without dissipation is exactly what had been described by Gill and Turner (1976). The effect is most pronounced in the band of relatively deep winter mixed layers extending from the northern Sargasso Sea in a northeastward direction. Here, the winter mixed layer deepens from about 300 $\mathrm{m}$ in experiment KTEXP to more than $500 \mathrm{~m}$ when implicit convection is used (KTIMP). When combined with a restoring type of formulation of surface heat and freshwater fluxes, the generally deeper (and colder) 

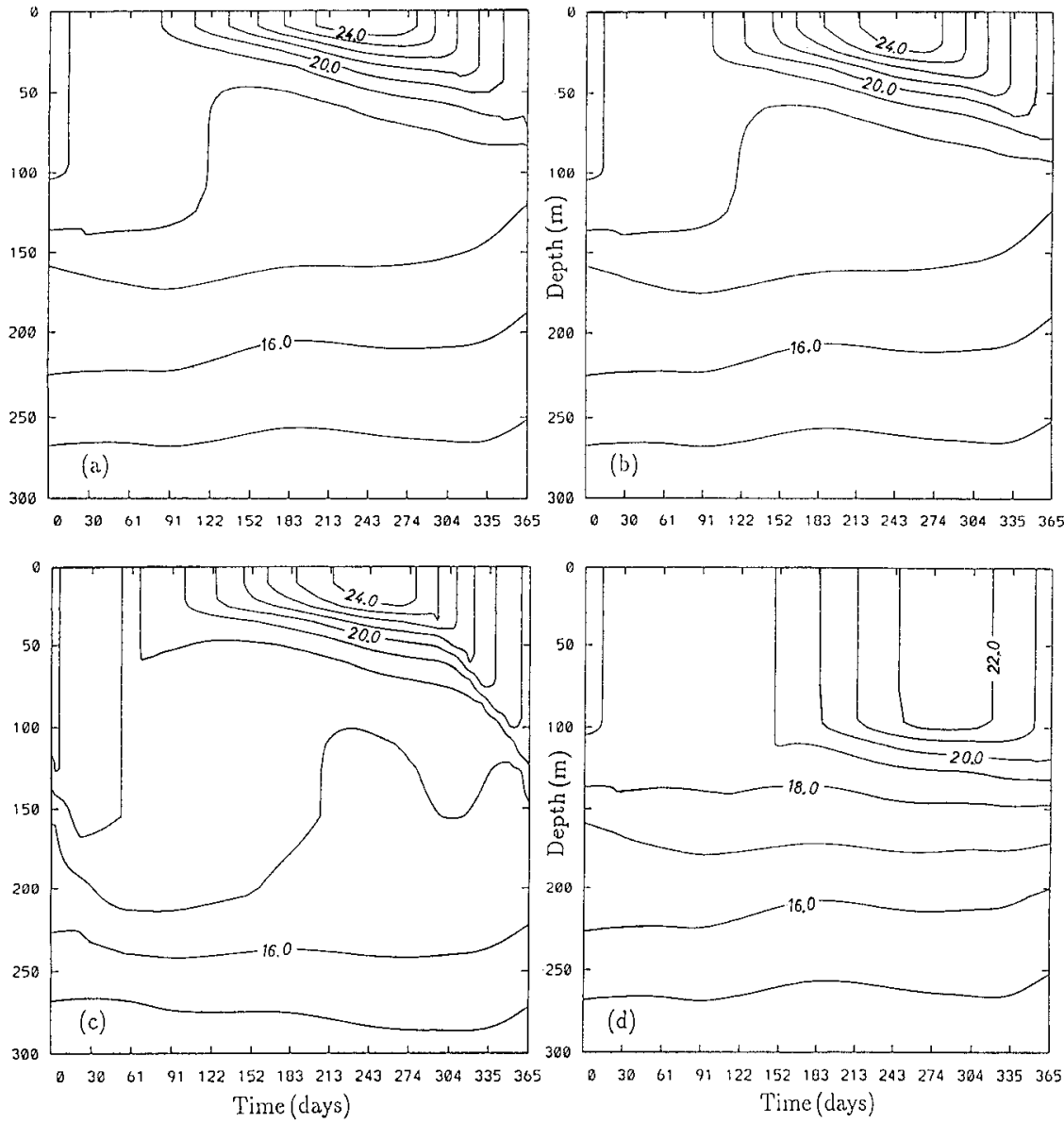

FIG. 1. Annual cycle of potential temperature over the upper $300 \mathrm{~m}$ at $30^{\circ} \mathrm{N}, 20^{\circ} \mathrm{W}$ : (a) experiment NOIMP without mixed layer, (b) experiment KTEXP with Kraus-Turner type mixed layer and explicit convective adjustment, (c) experiment KTIMP with Kraus-Turner type mixed layer and implicit convective adjustment $\left(K_{\rho}=10^{6} \mathrm{~cm}^{2} \mathrm{~s}^{-1}\right.$ if $\left.\partial \rho / \partial z>0\right)$, (d) experiment KTISO with KrausTurner type mixed layer and modified implicit convective adjustment $\left(K_{\rho}=10^{6} \mathrm{~cm}^{2} \mathrm{~s}^{-1}\right.$ if $\left.\partial \rho / \partial z \geq 0\right)$.

mixed layers lead to enhanced buoyancy input into the upper ocean. Because the mixed layer routine was not applied below a depth of $720 \mathrm{~m}$ (section 2), the additional buoyancy gain of the upper ocean can actually lead to a reduction in the depth of deep $(>720 \mathrm{~m})$ mixed layers. This explains, for example, the reduction in the area of deep winter convection in the Labrador Sea.

\section{2) Solution}

Obviously, the unintended simulation of fully penetrative convection arises from using a simplified KrausTurner model [Eq. (8)] that neglects the buoyancy terms in the TKE budget of the mixed layer. While this works well if the mixed layer routine is applied after an explicit convective adjustment scheme has removed all static instabilities, problems arise when convection is handled implicitly by the differential form of vertical diffusion. In this case, the mixed layer model itself must be able to accurately account for statically unstable situations.
This can be ensured by including the buoyancy terms in the TKE budget [i.e., use Eq. (5) instead of (8)] when implicit convective adjustment is employed. Such an experiment was run and found to produce virtually identical results to KTEXP.

Although the mixed layer routine employing Eq. (5) accurately removes all static instabilities arising from direct buoyancy loss at the surface, a convective adjustment routine is still necessary to remove instabilities in the ocean interior that are not directly connected to the surface mixed layer. Such instabilities may be produced well below the ocean surface by horizontal advection or diffusion of different water masses.

\section{b. Mixed layer model and isopycnal mixing}

\section{1) Problem}

A distinct problem arises when implicit vertical diffusion becomes large already for neutrally stable situ- 

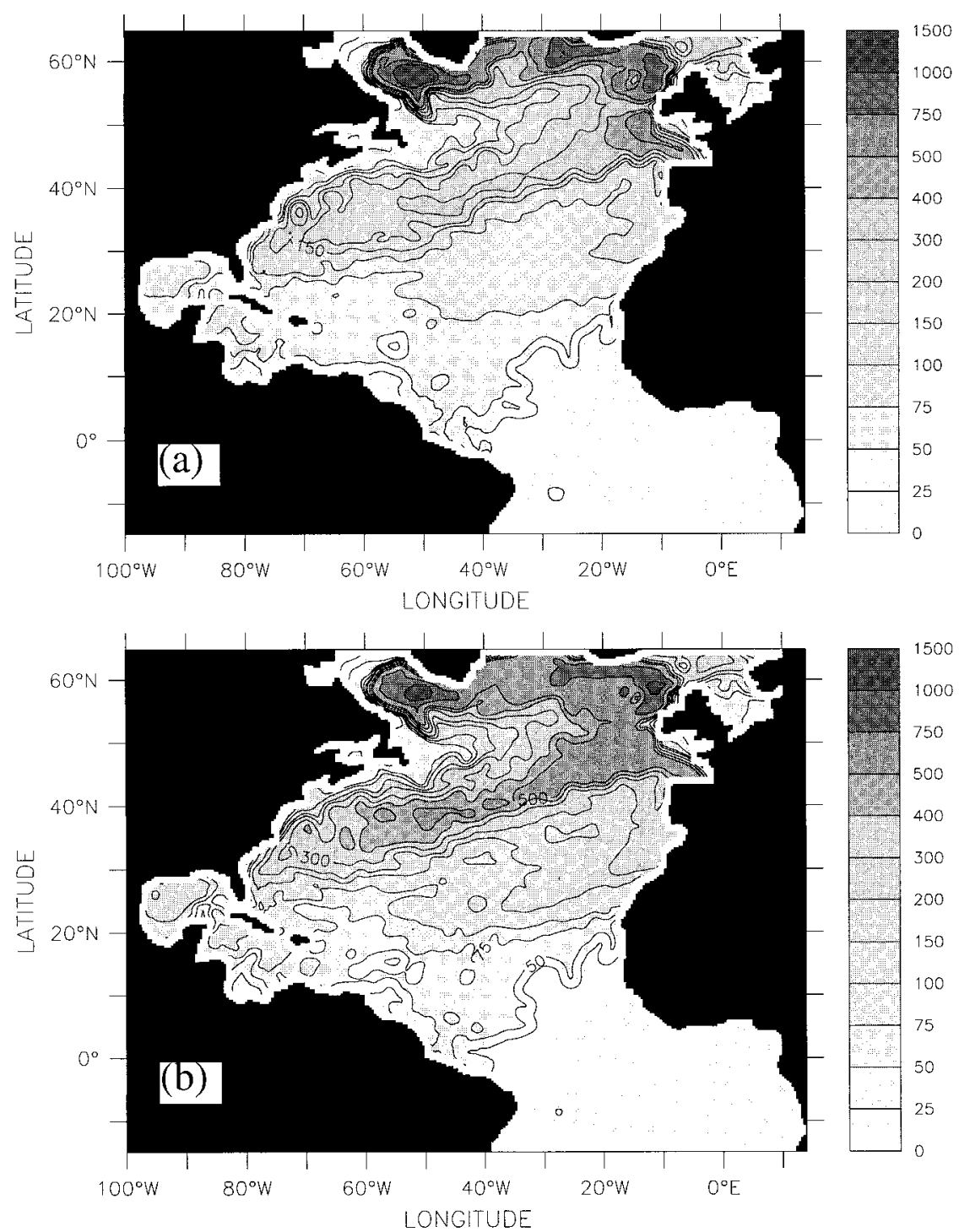

FIG. 2. Depth of the mixed layer at the beginning of March after $5 \mathrm{yr}$ of integration starting from Levitus (1982) temperature and salinity fields. The depth of the mixed layer is defined by a potential density interval of $0.01 \mathrm{~kg} \mathrm{~m}^{-3}$ with respect to the surface density: (a) experiment KTEXP, (b) experiment KTIMP.

ations. This would, for example, apply to common isopycnal mixing schemes (e.g., Redi 1982; Gent and McWilliams 1990) as long as isopycnal diffusion is not tapered to approach zero for extremely steep isopycnal slopes (e.g., Gerdes et al. 1991). In experiment KTISO we investigate the (extreme) case of switching to $K_{\rho}=$ $10^{6} \mathrm{~cm}^{2} \mathrm{~s}^{-1}$ for neutrally stratified situations. That is, implicit convection sets in already for $\partial \rho / \partial z=0$.

Figure 1d shows that winter mixed layer depths now are very close to those simulated by NOIMP and KTEXP. However, the capping of the deep winter mixed layer by a shallow warm surface layer in spring is almost completely suppressed. The reason for this poor performance is found in the time sequencing of the mixed layer scheme and implicit convection. Convection will now always reach at least as deep as the previous time step's mixed layer depth. In fact, stabilizing vertical density gradients that may accumulate over one time step will generally be mixed twice: first by the implicit vertical mixing routine, and then by the mixed layer scheme. Except for very strong stabilizing surface forcing and/or very small wind forcing the mixed layer routine can easily penetrate the weak stratification that is left after the action of the large $K_{\rho}$, resulting in $\partial \rho / \partial z$ $=0$ and again in convection at the following time step. Calling a Kraus-Turner type model after an implicit mixing scheme that increases vertical diffusion in neutrally stratified fluid will thus lead to systematically 
overestimated mixed layer depths in spring and summer, independently whether Eq. (8) or Eq. (5) is used.

\section{2) Solution}

One obvious solution to the above problem is to ensure that all mixing in neutrally stable situations is left to the mixed layer model, for example, by tapering vertical diffusivities of any differential mixing scheme to zero for $\partial \rho / \partial z=0$. Alternatively, the problem may be eliminated by calling the Kraus-Turner type mixed layer routine [using Eq. (5)] before the implicit vertical mixing routine. Stabilizing density fluxes will then be fully considered by the mixed layer model. Although the subsequent implicit vertical mixing may still mix farther down, it will generally result in a stably stratified-and thus nonconvective-water column, that is, $\partial \rho / \partial z<0$ at the end of a time step.

That this alternative time sequencing of mixed layer routine and convective adjustment works well is demonstrated by the following experiment:

- KTNEW: Kraus-Turner type wind- and buoyancyforced mixed layer [solving Eq. (5) with $n=0$ to simulate nonpenetrating convection], called before implicit convection. Convection already for neutrally stable situations, $K_{\rho}=10^{6} \mathrm{~cm}^{2} \mathrm{~s}^{-1}$ if $\partial \rho / \partial z \geq 0$.

Indeed, the results of KTNEW are virtually identical to those of KTEXP. For completeness, we note that essentially the same results were also obtained in an experiment with $K_{\rho}=10^{6} \mathrm{~cm}^{2} \mathrm{~s}^{-1}$ only if $\partial \rho / \partial z>0$ [i.e., KTNEW also solves the problem described in section $3 a(1)]$.

\section{Summary}

It has been demonstrated that the combination of different subroutines that separately account for different mechanisms of vertical mixing can introduce inconsistent mutual interactions arising from the sequential passing of information about stratification through the individual mixing schemes. The schemes considered may be divided into two classes: adjustment schemes that directly rearrange the current $T$ and $S$ fields without the intermediate calculation of diffusivities, and differential schemes that use finite diffusivities that are computed from the stratification at a previous time step. The first group consists of explicit convective adjustment and Kraus-Turner type mixed layer schemes, whereas in the second we have implicit convective adjustment as well as typical isopycnal mixing schemes.

In this note it is argued that special care has to be taken when a Kraus-Turner type mixed layer model is combined with any of the differential mixing schemes. One possible strategy that avoids unintended interactions between the different mixing routines has been presented in the form of experiment KTNEW. Here, the Kraus-Turner model, which now, even for the case of simulating wind-forced mixing, must account for buoyancy fluxes, is called before the various implicit mixing schemes, like implicit convective adjustment or isopycnal diffusion, are applied.

To what extent do the results reported here affect previous CME experiments with a Kraus-Turner type mixed layer that were set up like configuration KTIMP? The unintendedly simulated fully penetrating convection is generally associated with a reduction in surface temperature and an increase in the heat flux into the ocean. In our integration of both KTEXP and KTIMP over a 5-yr period (which is typical for most CME experiments), changes in surface heat flux were found to be largest over the northern part of the subtropical gyre where KTIMP absorbs up to $20 \mathrm{~W} \mathrm{~m}^{-2}$ more heat than experiment KTEXP. Averaged over the entire model domain and integration period, the heat flux of run KTIMP is only $2 \mathrm{~W} \mathrm{~m}^{-2}$ larger than in experiment KTEXP.

Given the relatively short integration times, differences in the density field between KTIMP and KTEXP remain relatively small. Although there is a slight change in water mass properties, particularly in the source region of subpolar mode waters, changes in circulation or heat transport are very small. Consequently, it is not expected that repeating previous CME experiments, but using configuration KTEXP instead of KTIMP, will significantly alter published results, except for figures that explicitly show the overestimated depth of the mixed layer (e.g., Fig. 10 of Oschlies and Willebrand 1996; Fig. 3 of Böning and Herrmann 1994). In fact, it was only the coupling of a pelagic ecosystem model (Oschlies and Garçon 1999) with its strong sensitivity to changes in the mixed layer depth that led to the identification of the previously unnoticed problem.

Acknowledgments. This study was supported through the European Union ESCOBA program (Contract ENV4-CT95-0132) and German JGOFS (Contract 03F0160D). The author thanks the reviewers for their very helpful and constructive comments.

\section{REFERENCES}

Böning, C. W., and P. Herrmann, 1994: On the annual cycle of poleward heat transport in the ocean: Results from high-resolution modeling of the north and equatorial Atlantic. J. Phys. Oceanogr., 24, 91-107.

Bryan, F. O., and W. R. Holland, 1989: A high resolution simulation of the wind and thermohaline-driven circulation in the north Atlantic Ocean. Parameterization of Small-Scale Processes, Proc. 'Aha Huliko'a, Hawaiian Winter Workshop, Hawaii Inst. Geophys. Spec. Publ., 99-115.

Camp, N. T., and R. L. Elsberry, 1978: Oceanic thermal response to strong atmospheric forcing. II. The role of one-dimensional processes. J. Phys. Oceanogr., 8, 215-224.

Gargett, A. E., 1986: Small-scale parameterization in large-scale ocean models. Advanced Physical Oceanographic Numerical Modelling, J. J. O'Brien, Ed., D. Reidel, 145-154.

Gent, P. R., and J. C. McWilliams, 1990: Isopycnal mixing in ocean circulation models. J. Phys. Oceanogr., 20, 150-155. 
Gerdes, R., C. Köberle, and J. Willebrand, 1991: The influence of numerical advection schemes on the results of ocean general circulation models. Climate Dyn., 5, 211-226.

Gill, A. E., and J. S. Turner, 1976: A comparison of seasonal thermocline models with observation. Deep-Sea Res., 23, 391-401.

Han, Y.-J., 1984: A numerical world ocean circulation model. Part II: A baroclinic experiment. Dyn. Atmos. Oceans, 8, 141-172.

Hellerman, S., and M. Rosenstein, 1983: Normal monthly wind stress over the World Ocean with error estimates. J. Phys. Oceanogr., 13, 1093-1104.

Kraus, E. B., and C. Rooth, 1961: Temperature and steady state vertical heat flux in the ocean surface layers. Tellus, 13, 231-238.

_ thermocline. II. The general theory and its consequences. Tellus, 19, 98-106.

Levitus, S., 1982: Climatological Atlas of the World Ocean. NOAA Prof. Pap. 13, U.S. Govt. Printing Office, 173 pp.

Marotzke, J., 1991: Influence of convective adjustment on the stability of the thermohaline circulation. J. Phys. Oceanogr., 21, 903907.

Niiler, P. P., and E. B. Kraus, 1977: One-dimensional models of the upper ocean. Modelling and Prediction of the Upper Layers of the Ocean, E. B. Kraus, Ed., Pergamon Press, 143-172.

Oschlies, A., and J. Willebrand, 1996: Assimilation of Geosat altimeter data into an eddy-resolving primitive equation model of the North Atlantic Ocean. J. Geophys. Res., 101, 14 175-14 190.

— and V. Garçon, 1999: An eddy-resolving coupled physicalbiological model of the North Atlantic. Part I: Sensitivity to physics and numerics. Global Biogeochem. Cycles, 13, 135-160.

Pacanowski, R. C., K. Dixon, and A. Rosati, 1991: The GFDL Modular Ocean Model users guide version 1.0. GFDL Ocean Group Tech. Rep. No. 2.

Rahmstorf, S., 1993: A fast and complete convection scheme for ocean models. Ocean Modeling (unpublished manuscripts), 101, 9-11.

Redi, M. H., 1982: Oceanic isopycnal mixing by coordinate rotation. J. Phys. Oceanogr., 12, 1154-1158.

Sterl, A., and A. Kattenberg, 1994: Embedding a mixed layer model into an ocean general circulation model of the Atlantic: The importance of surface mixing for heat flux and temperature. $J$. Geophys. Res., 99, 14 139-14 157.

Thompson, R. O. R. Y., 1976: Climatological numerical models of the surface mixed layer of the ocean. J. Phys. Oceanogr., 6, 496-503. 